\title{
FOXC2 disease-mutations identified in lymphedema-distichiasis patients cause both loss and gain of protein function
}

\author{
Daniela Tavian ${ }^{1}$, Sara Missaglia ${ }^{1}$, Paolo E. Maltese ${ }^{2}$, Sandro Michelini ${ }^{3}$, Alessandro \\ Fiorentino ${ }^{3}$, Maurizio Ricci ${ }^{4}$, Roberta Serrani ${ }^{4}$, Michael A. Walter ${ }^{5,6}$ and Matteo \\ Bertelli² $^{2}$ \\ ${ }^{1}$ Laboratory of Cellular Biochemistry and Molecular Biology, CRIBENS, Catholic University of the Sacred Heart, Milan, Italy \\ ${ }^{2}$ MAGI Non-Profit Human Medical Genetics Institute, Rovereto (TN), Italy \\ 3 Department of Vascular Rehabilitation, San Giovanni Battista Hospital, Rome, Italy \\ ${ }^{4}$ Medicina Riabilitativa, Azienda Ospedaliero-Universitaria Ospedali Riuniti di Ancona, Torrette, Italy \\ ${ }^{5}$ Department of Medical Genetics, University of Alberta, Edmonton, Alberta, Canada \\ ${ }^{6}$ Department of Ophthalmology and Visual Sciences, University of Alberta, Edmonton, Alberta, Canada \\ Correspondence to: Daniela Tavian, email: daniela.tavian@unicatt.it
}

Keywords: primary lymphedema, distichiasis, Foxc2 gene mutations, transcription factor, gain of function, Pathology Section Received: December 21, $2015 \quad$ Accepted: May 22, 2016

Published: June 02, 2016

\section{ABSTRACT}

Dominant mutations in the FOXC2 gene cause a form of lymphedema primarily of the limbs that usually develops at or after puberty. In $90-95 \%$ of patients, lymphedema is accompanied by distichiasis. FOXC2 is a member of the forkhead/ winged-helix family of transcription factors and plays essential roles in different developmental pathways and physiological processes. We previously described six unrelated families with primary lymphedema-distichiasis in which patients showed different FOXC2 mutations located outside of the forkhead domain. Of those, four were missense mutations, one a frameshift mutation, and the last a stop mutation. To assess their pathogenic potential, we have now examined the subcellular localization and the transactivation activity of the mutated FOXC2 proteins. All six FOXC2 mutant proteins were able to localize into the nucleus; however, the frameshift truncated protein appeared to be sequestered into nuclear aggregates. A reduction in the ability to activate FOXC1/FOXC2 response elements was detected in $50 \%$ of mutations, while the remaining ones caused an increase of protein transactivation activity. Our data reveal that either a complete loss or a significant gain of FOXC2 function can cause a perturbation of lymphatic vessel formation leading to lymphedema.

\section{INTRODUCTION}

Lymphedema is a dysfunction of the lymphatic system, a disorder characterized by abnormal swelling of one or more extremities due to impaired transport of the lymph [1-3]. This common and debilitating condition affects millions of people worldwide. Lymphedema can be primary (congenital) or secondary (acquired). The prevalence of primary lymphedema has been estimated at 1-5 per 10000 persons (http://www.orpha.net). More than 10 Mendelian forms are known, of which three are considered the major forms: Milroy disease (MD) [MIM 153100], lymphedema-distichiasis syndrome (LDS) [MIM 153400] and Meige disease (MGD) [MIM 153200] [4, 5].
Dominant mutations in the FOXC2 gene (MIM 602402), coding for the forkhead transcription factor $F O X C 2$, cause a form of lymphedema with variable age of onset (range: 7-40 years), often associated with distichiasis [6-8]. FOXC2 plays a key role in regulation of lymphatic endothelial cells differentiation, in formation of smooth muscle cell layers and in morphogenesis of lymphatic valves. FOXC2 and VEGFR-3 act through a common genetic pathway to establish distinct properties of the lymphatic vascular architecture [9].

FOXC2 maps to $16 \mathrm{q} 34.3$ and it produces a $2.2 \mathrm{~kb}$ transcript with a $1.5 \mathrm{~kb}$ single exon coding region The FOXC2 protein contains 501 amino acids (Figure 1). The most characterized region in the gene is the forkhead DNA 
binding domain (FHD, amino acids 71 to 162), containing also the nuclear signal 1 (NLS1, amino acids 78-93). At the N-terminal there is the transactivation domain 1 (AD1) starting from the first amino acid until the FHD (amino acid 71). In the central region of FOXC2 protein, after the NLS2 (amino acids 168-176), some phosphorylation and SUMOylation conserved sites have been recently identified, conferring to this sequence a negative regulative role $[10,11]$. Finally, in the C-terminal sequence, a second transactivation domain has been described (AD-2, amino acids 395-494) and an inhibitory region (ID-2, amino acids 495-501) [10, 12].

In LD patients, almost 70 different $F O X C 2$ mutations have been reported to date, scattered all along the coding sequence (www.hgmd.cf.ac.uk). The majority of FOXC2 mutations are small insertions or deletions and nonsense mutations causing truncated proteins, which have been hypothesized to be responsible for a haplo-insufficiency condition $[7,8]$. The FOXC2 haplo-insufficient state is associated to hyperplasia and distichiasis in mice [13]. FOXC2-/- mice have been found to exhibit abnormal pericyte recruitment to the lymphatic capillaries and valve agenesis in the collecting vessels, indicating that FOXC2 is essential for the formation of a pericyte-free lymphatic network and lymphatic valve development [14]. Moreover, it was noted that the craniofacial, cardiovascular, and skeletal abnormalities sometimes associated with lymphedema-distichiasis syndrome in humans, had previously been shown to be fully penetrant in homozygous $F O X C 2$-null mice $[15,16]$.

The pathogenesis of LD was assumed to be associated with FOXC2 haplo-insufficiency also in humans, until some $F O X C 2$ missense mutations, identified in LD patients, were found to cause a gain of protein function [17]. Approximately $24 \%$ of $F O X C 2$ variations are missense mutations of which very few have been functionally investigated. Two mutations located inside the forkhead domain impaired the DNA-binding and transcriptional activation ability of FOXC2 protein [18]; in contrast, four mutations located outside the forkhead domain caused a gain of function [17]. Moreover, no data are available on the transactivation properties-function of FOXC2 truncated proteins due to stop mutations or small insertions/deletions preserving at least half of the protein sequence, although together they represent almost 50\% of $F O X C 2$ variations. We therefore performed functional characterization of $F O X C 2$ mutations located in different protein domains to provide novel information on structurefunction relationships of the FOXC2 transcription factor and to get further insight into the disease-causing mechanism of lymphedema.

\section{RESULTS}

\section{Patient descriptions}

Genetic evaluation of LD patients has been previously reported [19]. Molecular and clinical findings are shown in Table 1. All but two of the patients (P1 and P3) presented with distichiasis. No evidence of heart defects, cleft palate, extradural cysts or other distinctive characters have been reported, with the exception of P4. Finally, no superficial and deep venous insufficiency and recurrent erysipelas have been observed. A detailed clinical description of LD patients is reported in Supplementary material 1.

\section{Functional characterization of FOXC2 mutations}

The mutations analysed in this study were all localized outside the forkhead domain, in different regions of FOXC2 protein (Figure 1). The evaluation of missense mutations by bio-informatic prediction tools and the homology comparison between different species are reported in Supplementary Figure 1. To verify whether these mutations can affect FOXC2 function by reducing mRNA or protein stability, altering nuclear localization, or impairing transactivation activity, we subcloned wildtype $F O X C 2$ cDNA into the pcDNA3.1/NT-GFP-TOPO TA expression vector and then performed site-direct mutagenesis to generate the $F O X C 2$-GFP plasmids with the disease causing mutations.

When transfected into HeLa cells, wild type and mutant plasmids showed similar and stable FOXC2 mRNA levels (Figure 2A). Exogenous FOXC2 expression was detected by RT-PCR analysis with primers that selectively amplify the cDNA obtained from the recombinant plasmids. Endogenous GAPDH RT-PCR products were detectable in similar amounts in all samples tested and were used to normalize FOXC2 RT-PCR values for each sample (Figure 2B).

In agreement with RT-PCR results, western blotting analysis showed stable expression of all recombinant proteins (Figure 2C), that appeared to be phosphorylated with the only exception being p.M276DfsX186. There were no apparent differences in phosphorylation between wild type and FOXC2 proteins carrying missense mutations as detected in electrophoretic mobility assays. The Q420X FOXC2 protein was also phosphorylated, however it presented a different migration profile in comparison with wild type protein due to the mutation resulting in a truncated FOXC2 protein. Finally, the p.M276DfsX186 FOXC2 protein was detected as a single band on western blots, and thus appears not to be phosphorylated. The p.M276DfsX186 FOXC2 protein is 
Table 1: Summary of patients' molecular and clinical data

\begin{tabular}{|c|c|c|c|c|c|c|c|c|}
\hline Patient & Mutation & $\begin{array}{l}\text { Protein } \\
\text { domain }\end{array}$ & $\begin{array}{l}\text { Transcriptional. } \\
\text { activity of } \\
\text { mutated } \\
\text { protein* }\end{array}$ & $\begin{array}{l}\text { Age of } \\
\text { onset }\end{array}$ & Lymphedema & $\begin{array}{l}\text { Lymphatic } \\
\text { hypoplasia or } \\
\text { hyperplasia** }\end{array}$ & Distichiasis $* * *$ & $\begin{array}{l}\text { Cardiac } \\
\text { defects/ } \\
\text { other } \\
\text { clinical } \\
\text { findings }\end{array}$ \\
\hline $\begin{array}{l}1 \mathrm{M} \\
(69 \mathrm{y})\end{array}$ & A3G & AD-1 & $10 \%$ & $50 \mathrm{y}$ & $\begin{array}{l}\text { bilateral, } \\
\text { greater } \\
\text { right }\end{array}$ & hyperplasia & no & no \\
\hline $\begin{array}{l}2 \mathrm{M} \\
(46 \mathrm{y})\end{array}$ & M276fs & $\begin{array}{l}\text { Central } \\
\text { region }\end{array}$ & $16 \%$ & $12 \mathrm{y}$ & $\begin{array}{l}\begin{array}{l}\text { bilateral, } \\
\text { greater } \\
\text { right }\end{array} \\
\end{array}$ & hyperplasia & yes & no \\
\hline $\begin{array}{l}3 \mathrm{~F} \\
(19 \mathrm{y})\end{array}$ & S370T & $\begin{array}{l}\text { Central } \\
\text { region }\end{array}$ & $73 \%$ & $14 \mathrm{y}$ & bilateral & $\begin{array}{l}\text { uncertain } \\
\text { evaluation }\end{array}$ & no & no \\
\hline $\begin{array}{l}4 \mathrm{M} \\
(28 \mathrm{y})\end{array}$ & G420X & AD-2 & $257 \%$ & $19 y$ & $\begin{array}{l}\text { bilateral, } \\
\text { greater on } \\
\text { right }\end{array}$ & hypoplasia & yes & \begin{tabular}{|l|} 
Bicuspid \\
aortic \\
valve/ \\
extradural \\
cyst \\
\end{tabular} \\
\hline $\begin{array}{l}5 \mathrm{~F} \\
(34 \mathrm{y})\end{array}$ & L487P & AD-2 & $172 \%$ & $26 y$ & $\begin{array}{l}\text { bilateral, } \\
\text { greater on left }\end{array}$ & hypoplasia & yes & no \\
\hline $\begin{array}{l}6 \mathrm{M} \\
(30 \mathrm{y})\end{array}$ & A492V & AD-2 & $182 \%$ & $26 y$ & left unilateral & hypoplasia & yes & no \\
\hline
\end{tabular}

* The percentage of transcriptional activity of FOXC2 mutated proteins was calculated considering FOXC2 wild type signal as $100 \%$ of activity (Luciferase Reporter assay)

** Lymphatic hypoplasia or hyperplasia was defined by lymphoscintigraphy evaluation performed by two clinicians independently, referring on lymph nodes up take of radiocolloid and on the presence or absence of dermal backflow

*** Patients were examined by an ophthalmologist using a slit lamp for evidence of distichiasis

M: Male; F: Female

larger than the p.Q420X mutant protein, since the former consists of 462 amino acids (276 of FOXC2 protein plus 186 due to the frameshift) while the latter of 420 amino acids.

To assess whether mutations affected the ability of FOXC2 proteins to localize properly into the nucleus,
HeLa cells were transfected with wild type and mutant plasmids. We included also the FOXC2(R121H) mutationcontaining plasmid as a control in order to allow an accurate comparison of intracellular distribution, since the FOXC2(R121H) protein has been previously demonstrated to display a defect in nuclear localization, with partial

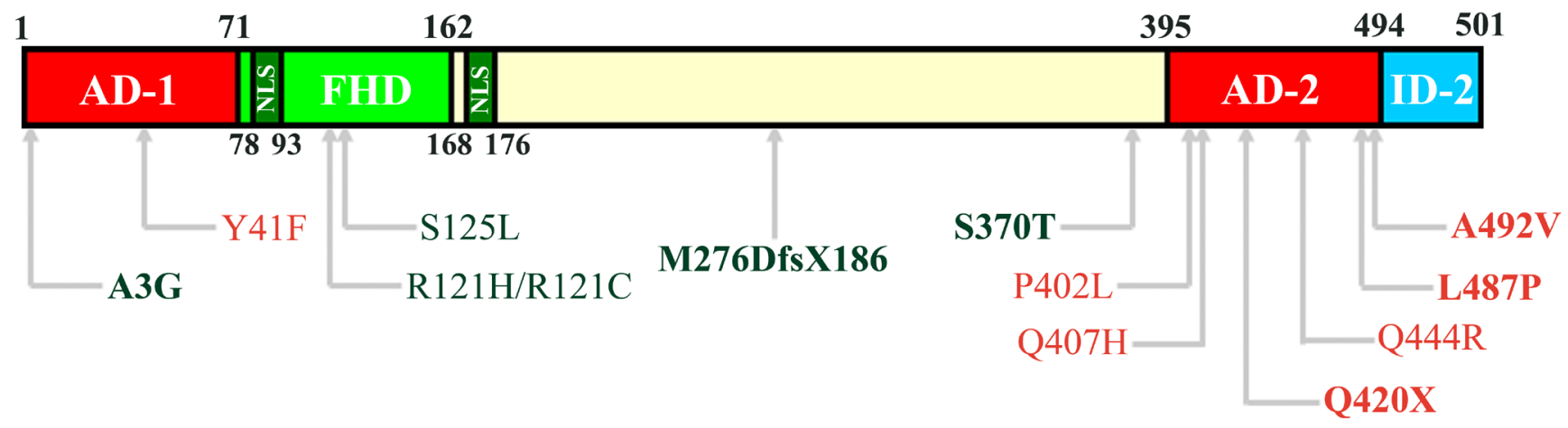

Figure 1: Structural domains of FOXC2 protein. In the schematic representation of FOXC2 (amino acids 1-501), the Activation Domain 1 (AD-1) is located between amino acid 1 and 71. The Forkhead Domain (FHD, amino acids 71-162) is the DNA-binding region and contains also the first Nuclear Signal (NLS, amino acid 78-93). The second NLS is located between amino acid 168 and 176. In the C-terminal region, there are the Activation Domain 2 (AD-2, amino acids 395-494) and the Inhibitory Domain 2 (ID-2, amino acids 494501). Furthermore, the localization of mutations analyzed by functional studies are reported in the scheme [Red: activating mutations; Green: inactivating mutations; Bold, mutations described by Michelini et al. (2012); Regular, mutations described by Berry et al. (2005) and van Steensel et al (2009)]. 
cytoplasmic signal [18]. As expected, immunofluorescence analysis revealed that wild type FOXC2 was localized exclusively into the nucleus and displayed a homogeneous distribution (Figure 3A). All disease mutations did not affect FOXC2 nuclear localization; moreover, no cytoplasmic signal could be detected for any of them (Figure 3A; Table 2). However, the transfection of FOXC2(M276DfsX186) recombinant plasmid caused the production of nuclear aggregates (Figure 3B). A similar effect was observed utilizing COS7 cells (Supplementary Figure 2). Also the $F O X C 2(\mathrm{R} 121 \mathrm{H})$ plasmid, in our system, induced intranuclear protein aggregation (Figure $3 \mathrm{~B})$. In contrast, the FOXC2(M276X) truncated protein revealed a homogeneous fluorescence signal, very similar to that of wild type (Figure 3B).

FOXC2 is known to act as a transcription factor; therefore, the ability of FOXC2 mutant proteins to stimulate the expression of a reporter gene was tested in HeLa and COS7 cells. GFP-FOXC2 fusion proteins recapitulated the transcriptional activity of FOXC2 mutant proteins expressed without an exogenous tag, in both HeLa and COS7 cells (Figure 4A and 4B). The Q420X

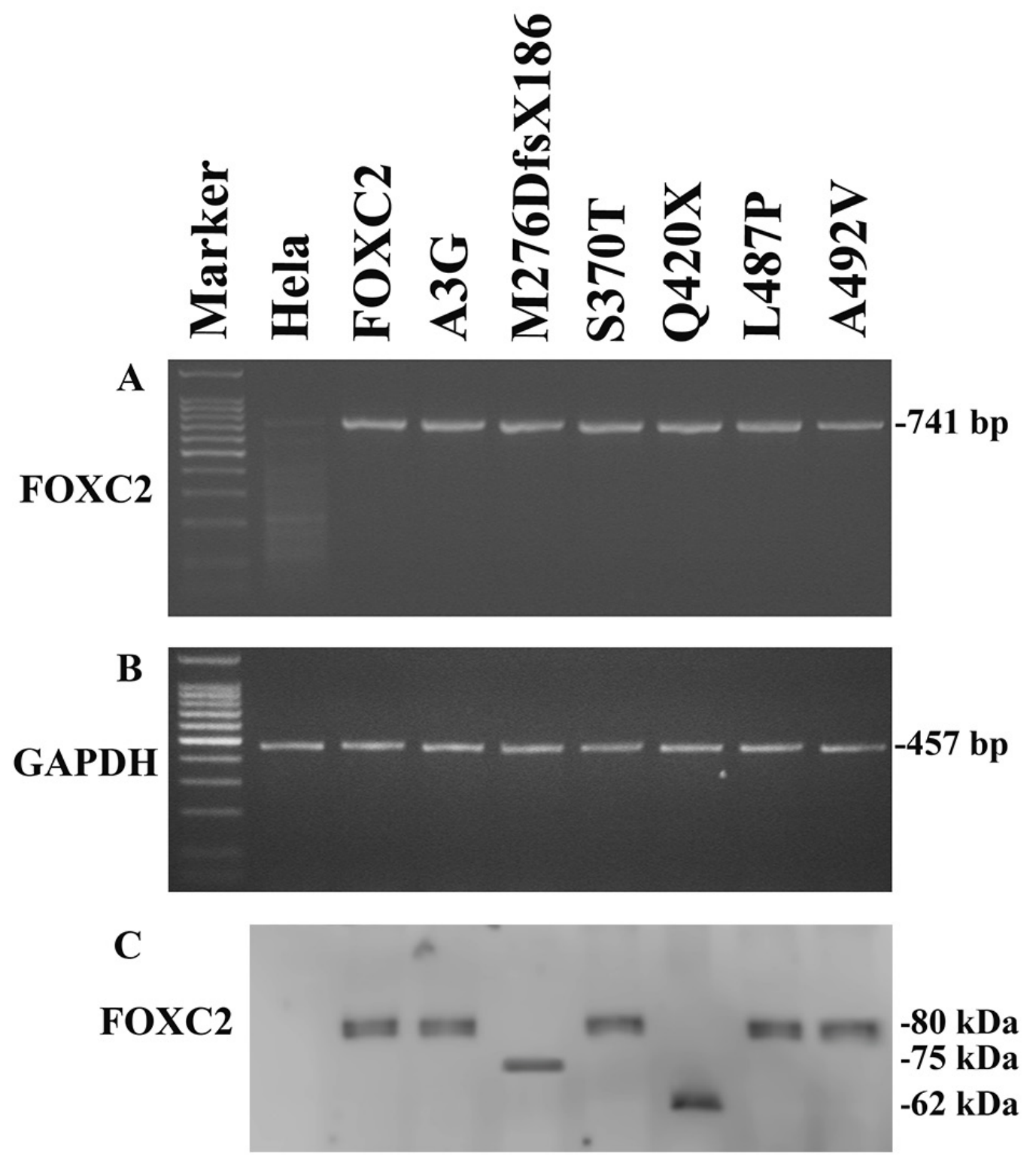

Figure 2: Exogenous FOXC2 expression of mutant recombinant plasmids. A. RT-PCR detection of exogenous FOXC2 (741-bp product) and B. GAPDH (457-bp product) in HeLa transfected cells with pFOXC2-GFP, pFOXC2(A3G)-GFP,pFOXC2(M276DfsX186)-

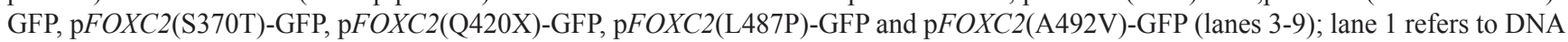
molecular weight marker and lane 2 refers to non-transfected HeLa cells. C. Western blotting analysis of exogenous FOXC2 protein expression in HeLa cells transfected with plasmids reported in (A) and in (B). 

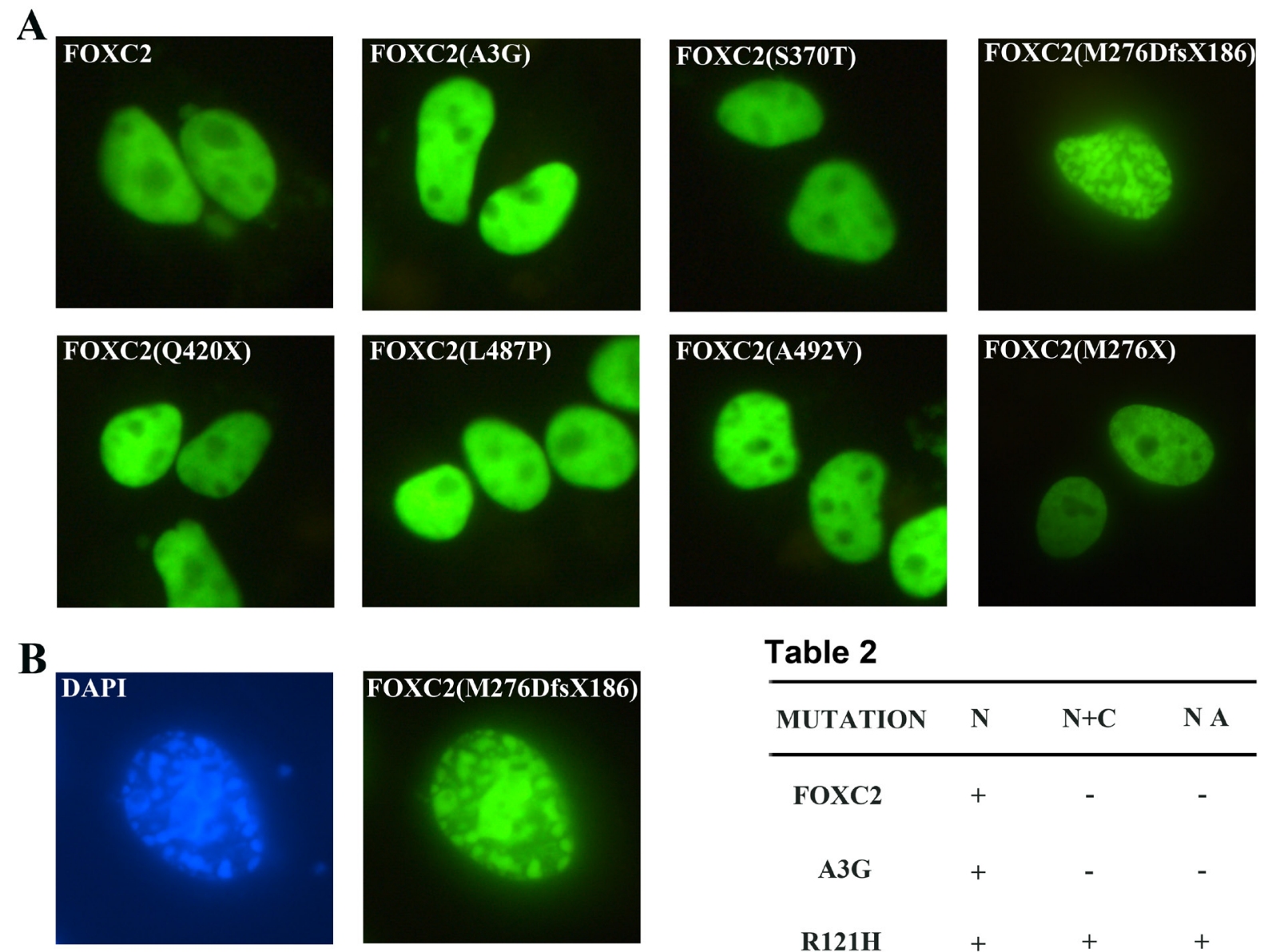

Table 2
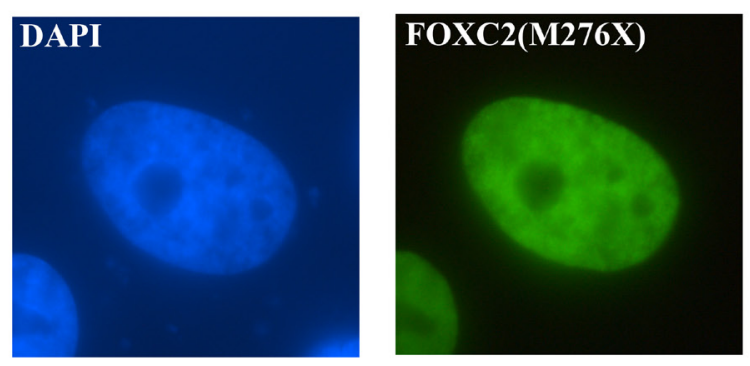

\begin{tabular}{cccc}
\hline MUTATION & N & N+C & N A \\
\hline FOXC2 & + & - & - \\
R121H & + & - & - \\
M276DX186 & + & - & + \\
M276X & + & - & - \\
S370T & + & - & - \\
Q420X & + & - & - \\
L487P & + & - & - \\
A492V & + & - & -
\end{tabular}
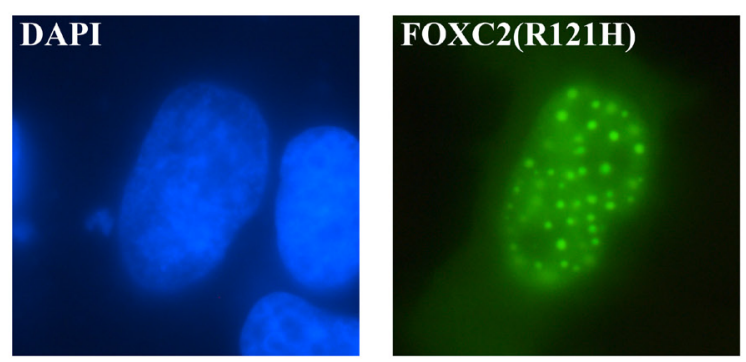

$\mathrm{N}$ : nuclear localization

$\mathrm{N}+\mathrm{C}$ : nuclear and citoplasmatic localization $\mathrm{N}$ A: nuclear aggregates

Figure 3: Transient transfection of FOXC2 mutant proteins in HeLa cells. HeLa cells were transiently transfected with one of the following plasmids: $\mathrm{p} F O X C 2-\mathrm{GFP}, \mathrm{p} F O X C 2(\mathrm{~A} 3 \mathrm{G})-\mathrm{GFP}, \mathrm{p} F O X C 2(\mathrm{~S} 370 \mathrm{~T})-\mathrm{GFP}, \mathrm{p} F O X C 2(\mathrm{M} 276 \mathrm{Dfs} 186)-\mathrm{GFP}, \mathrm{p} F O X C 2(\mathrm{Q} 420 \mathrm{X})-$

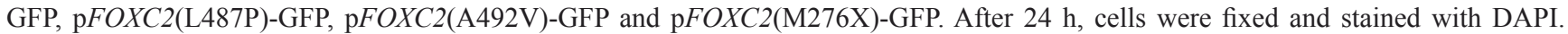
Fluorescence of FOXC2-GFP fusion proteins is in green. A. The nuclear localization of all FOXC2 mutant proteins was detected by direct immunofluorescence analysis of plasmids tagged with GFP; images were at 40X magnification. B. Immunofluorescence evaluation of HeLa cells transiently transfected with the following recombinant plasmids: $\mathrm{p} F O X C 2(\mathrm{M} 276 \mathrm{DfsX} 186), \mathrm{p} F O X C 2(\mathrm{M} 276 \mathrm{X})$ and $\mathrm{p} F O X C 2(\mathrm{R} 121 \mathrm{H})$. The M276DfsX186 mutation causes FOXC2 protein aggregates, as well the R121H mutation. In green, FOXC2 proteins tagged with GFP and in blue, cellular nuclei stained with DAPI. More than 200 cells were counted to detect nuclear versus cytoplasmatic localization. Magnification:100X. 

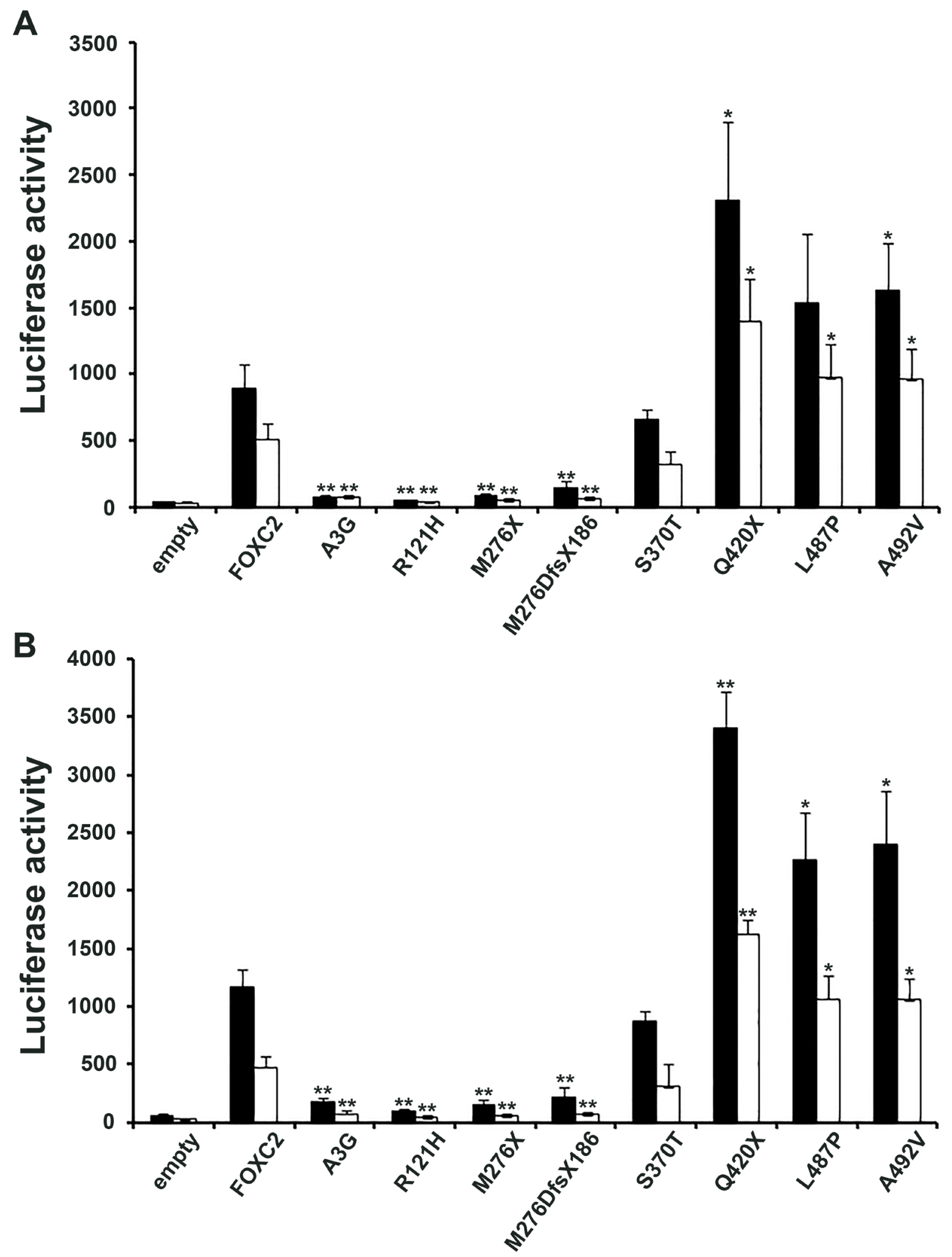

Figure 4: Transactivation activity of wt and mutant FOXC2 proteins. Luciferase assays were used to measure protein transactivation abilities. A. Transactivation activity of wt and mutant FOXC2-GFP fusion proteins compared with that of empty vector in HeLa (black squares) and COS7 (white squares) cells indicated as fluorescence units (vertical axis). B. Transactivation activity of wt and mutant FOXC2 proteins (proteins without any tag) compared with that of empty vector in HeLa or COS7 cells. Thick bar: mean value, error bar: SD. Significant differences were detected by using Student's t-test. P-values of $<0.05$ and of $<0.01$ were considered to be significant and indicated with "**"and "**”, respectively. 
and A492V mutations displayed significantly increased FOXC2 transcriptional activity in comparison with wild type protein in all the experimental conditions tested (Figure 4). The L487P mutation showed significance significant gain of protein function in 3 out 4 of luciferase assays. In contrast, the A3G and M276DfsX186 mutations were not able to activate the luciferase reporter vector above background levels (Figure 4). These mutations appeared to be similar to $\mathrm{R} 121 \mathrm{H}$ variation, as they rendered the FOXC2 transcription factor totally inactive. Finally, the S370T mutation also decreased transcriptional activity but only to almost $30 \%$ of that of FOXC2 wild type protein (Figure 4). Again, mutation R121H served as methodological negative control in our experiment, since it had previously found to be transcriptionally inactive in luciferase assays [17].

\section{DISCUSSION}

Despite the great importance of the FOXC2 transcription factor in human pathophysiology and the increasing number of different mutations identified in patients with lymphedema, the molecular consequences caused by $F O X C 2$ gene variations are almost entirely unknown [20-22]. Only a small percentage of FOXC2 mutations have been functionally investigated so far $(7 / 67$, $\sim 10 \%$ ); all of which are missense mutations $[17,18]$. In this report, we have analyzed the functional consequences of six FOXC2 disease mutations identified in LD patients. The A3G mutation is the FOXC2 mutation closest to the ATG starting codon identified to date. The p.A3G variation was considered "probably damaging" by Polyphen-2 and "deleterious" by Sift. This mutation is localized in the highly conserved AD-1 region of the FOXC2 protein. Although the $\mathrm{A} 3 \mathrm{G}$ mutation does not change the amino acid charge, both bioinformatics prediction and our functional data reveal that it is a pathogenic mutation, drastically reducing FOXC2's capability to activate expression of target genes. It is difficult to explain the biological dysfunction caused by single site amino acid replacement that does not result in charge-changing, unless it is assumed that this mutation decreases the ability to adopt the FOXC2 wild type conformation. As the complete $3 \mathrm{D}$ structure of FOXC2 protein is currently not available, it is not currently possible to exam the $3 \mathrm{D}$ structure of the $\mathrm{A} 3 \mathrm{G}$ mutant protein. Interestingly, a significant increase of function was previously reported in FOXC2 protein carrying the p.Y41F mutation, also located within the AD-1 domain [17]. Taken together, these results indicate that missense mutations within the FOXC2 AD-1 can result in either the loss or gain of function.

We examined three FOXC2 mutations located within the $\mathrm{AD}-2$ region: two missense (p.L487P and p.A492V) and one nonsense (p.Q420X) mutations. All three AD-2 mutations significantly increased FOXC2 transcriptional ability. Surprisingly, the p.Q420X displayed the highest level of activation, despite the truncated protein lacked more than half of AD-2 sequence and the entire ID-2 region. Consistent with these findings, Van Steensel et al. also reported a gain of function for FOXC2 missense variations localized inside AD-2 domain. We did not detect any evidence of mRNA or protein stability alterations resulting from FOXC2 AD-2 mutations. Thus, it is possible that AD-2 mutations exert their influence by modifying the tridimensional structure of the C-terminal domain of the FOXC2 protein and/or changing the ability of FOXC2 to interact with regulatory proteins. In the central region of $F O X C 2$, we have identified the p.M276DfsX186 mutation (in Patient 2) which leads to the formation of a truncated protein lacking of 235 amino acids in the C-terminal region, and with the addition of 186 nonsense amino acids due to the frameshift (Figure 1). This mutated protein did not have an impaired ability to localize into the cell nucleus, however, the FOXC2 p.M276DfsX186 mutant protein condensed into protein aggregates. In contrast, the p.M276X FOXC2 mutation localized to the nucleus without aggregating. We hypothesize that the presence of the extra 186 nonsense amino acids due the frameshift mutation resulted in the protein aggregation. Never the less, both p.M276DfsX186 and p.M276X FOXC2 mutations caused the complete loss of protein function, as might be expected for mutations that result in mutant proteins lacking about one half of the FOXC2 protein.

Finally, the S370T missense mutation, located in the terminal sequence of central region (Figure 1), resulted in a slight decrease of the ability of FOXC2 to trans-activate genes. Interestingly, S367 has been recently identified as a phosphorylation site for FOXC2 [11]. It thus cannot be excluded that S370T mutation partially influences the phosphorylation process of S367, triggering changes in the FOXC2 transcriptional program.

To date, it is not possible to extrapolate general conclusions about the relationship between FOXC2 activity and the protein variation position, because of the very limited number of different types of mutations (missense, nonsense and frameshift) that have been functionally tested. However, data obtained so far indicate that missense mutations in FHD and AD-2 domain might represent an exception, causing always a loss and a gain of FOXC2 function, respectively. More data are needed to highlight possible correlation between mutation position and function in other FOXC2 protein domains.

Nowadays, it is possible to estimate the theoretical pathogenic effects of missense mutations utilizing bioinformatics tools. However, the usefulness of these programs in predicting the real contribution of a mutation becomes limited when they provide conflicting data (see Supplementary Figure 1B, for p.A492V mutation) [23]. Moreover, current bioinformatics tools are not able to predict whether a mutation causes a gain or a loss of function. Only functional studies indicate how 
mutations impair protein ability, providing data that could be clinically relevant. This is true for genes encoding metabolic enzymes critically important for human pathologies [24-27] and, even more, for transcription factors, like $F O X C 2$, that are responsible for regulating the expression of a broad range of genes both during development and in adult tissues [12, 17, 28].

From our data it appears that no easy relationships can be established between the level of transcriptional activity of FOXC2 mutated proteins and the degree of pathology. Never the less, some clinically relevant information appears to be revealed by our analyses. After comparing the lymphoscintigraphy of the two patients harboring a considerable loss of function mutations (P1 and P2) with the others carrying a gain of function mutations (P4, P5 and P6), we noted some differences in the uptake of the radiopharmacon by inguinal lymph glands and its reflux back down into the lower leg. In particular, P1 and P2 patients showed a lymphoscintigram profile/images compatible with a hyperplasia of lymphatic vessels (with dermal backflow), while P4, P5 and P6 patients reports resembled more a hypoplastic condition (Supplementary Fig 3). While, lymphangiography is the best method to distinguish between hyper and hypoplasia $[8,17]$, our investigation indicate that lymphoscintigraphy may provide additional indication in those cases in which a considerable difference of gain or loss of function can be detected. In patient P3, where, a mild hyperplasia might be predicted since the S370T mutation causes a slight loss of transcriptional activity, it was not possible to highlight the modest increase of new lymphatic vessel formation using lymphoscintigraphy.

Based upon clinical and molecular data, therefore, we can hypothesize that an increased activity of FOXC2 results in disregulated expression of proteins essential for normal development of lymphatic system, causing hypoplasia. In contrast, decreased FOXC2 transcriptional activity appears to be associated with a hyperplastic condition. Our hypothesis on hypo or hyperplasia and activating versus inactivating mutations is consistent with previously reported results $[17,18]$ and with the hyperplastic condition of haplo-insufficienct FOXC2+/mice who present with abnormal lymphatic drainage, increased number of lymph nodes and lymph backflow $[13,14]$. Should transgenic mice carrying FOXC2 activating mutations become available, it would be interesting to discover if they develop a hypoplastic lymphatic system as predicted by our model.

The association of $\mathrm{FOXC2}$ mutation function and distichiasis is less clear however. Our patients with activating mutations presented with distichiasis, in contrast, 2 out of 3 patients with inactivating mutation did not. From these clinical findings, it might be tempting to speculate that distichiasis is associated with hypoplasia rather than hyperplasia. However, additional larger clinical studies are required to definitely elucidate correlations between $F O X C 2$ different mutations and the morphologic changes of lymphatic system, and with distichiasis.

In conclusion, we have analyzed the molecular consequence of FOXC2 mutations identified in six Italian families with lymphedema-distichiasis, providing evidences of patients with inactivating versus activating mutations of the FOXC2 transcription factor. During development, transcriptional programs of lymphatic endothelial cells are established by networks of transcriptional factors, among which FOXC2 is one of the most important. FOXC2 acts downstream of VEGFR-3 signaling pathway and is essential for the formation of pericyte-free lymphatic network. Moreover FOXC2 regulates the expression of genes essential for all steps of lymphatic valve morphogenesis and maintenance, such as connexins CX43 and CX47 [4, 5, 29, 30]. Our data indicate that either loss or gain of FOXC2 function is deleterious, causing hyperplasia or hypoplasia respectively in patients with primary lymphedema-distichiasis. We predict that any change in the activity level of FOXC2 causes an unbalanced expression of molecular regulators of lymphangiogenesis, a highly orchestrated process not yet fully understood.

Finally, recent studies show that FOXC2 overexpression is involved in cancer progression, inducing epithelial mensenchymal transition (EMT) [31, 32]. LDS patients carrying FOXC2 gain of function mutations should be carefully monitored in order to verify whether they present an higher cancer susceptibility and to ensure the earliest identification of possible lesions.

\section{MATERIALS AND METHODS}

\section{Cloning the $F O X C 2$ cDNA and generation of site- directed mutagenesis plasmids}

FOXC2 cDNA was amplified by PCR using DNA clone ID 32938 (Thermo Scientific) as template and the following primers: FOXC2-F 5'-ATGCAGGCGCGCTACTCC-3' and FOXC2-R 5'-TCAGTATTTCGTGCAGTCGTAGGAG-3'. The PCR product was subcloned into pcDNA3.1/NT-GFP-TOPO from Invitrogen to produce NT-GFP-FOXC2, expressing FOXC2 with GFP at the N-terminus. Point mutations were performed using the Phusion Site-Directed Mutagenesis Kit (Thermo Scientific). Mutations in FOXC2 cDNA were introduced using the following primers: $A 3 \mathrm{G}$ forward 5'-ATGCAGGGGCGCTACTCCGTGT-3' and reverse 5'-ACACGGAGTAGCGCCCCTGCAT-3'; R121H forward 5' GCAGAACAGCATCCACCACAACCTCTCGC -3' and reverse 5 ' GCGAGAGGTTGTGGTGGATGCTGTTCTGC $-3^{\prime}$; S370T forward 
5'-GTCGCCCCTGACCGCTCTCAACC-3' and reverse 5'-GGTTGAGAGCGGTCAGGGGCGAC -3'; Q420X forward 5'- GCCGCGGCGTAGGCGGCCT-3' and reverse 5'- AGGCCGCCTACGCCGCGGC -3'; L487P forward 5'-ACGCCGCCTCCCTATCGCCAC-3' and reverse 5'-GTGGCGATAGGGAGGCGGCGT-3'; A492V forward 5'-TCGCCACGCAGTCCCCTACTCCT-3' and reverse 5'-AGGAGTAGGGGACTGCGTGGCGA-3'.

In order to obtain the c.826-827delAT FOXC2 mutation (M276DfsX186) were used the following two couple of primers and two rounds of mutagenesis (see supplementary materials): forward(1) 5'CAGCGTGGAGAACATCTGACCCTGCGAACGTC-3' and reverse(1) 5'GACGTTCGCAGGGTCAGATGTTCTCCACGCTG-3' and forward(2) 5'CAGCGTGGAGAACATCGACCCTGCGAACGTC-3' and reverse(2) 5'GACGTTCGCAGGGTCGATGTTCTCCACGCTG-3'.

NT-GFP-FOXC2 (wild type) and NT-GFP-FOXC2 mutant plasmids were used as template to amplify FOXC2 control and mutants cDNA sequences with FOXC2-F and FOXC2-R primers (reported above). The PCR products were subcloned into pcDNA3.3-TOPO (Life Technologies) to produce control or mutant FOXC2 proteins without any tag. All final expression constructs were sequenced to verify that no additional mutations were introduced.

\section{RT-PCR analysis of FOXC2 recombinant mRNAs in HeLa cells}

HeLa cells were transiently transfected with NTGFP-FOXC2 recombinant plasmids using the TurboFect transfection reagent, according to the manufacturer's protocol (Thermo Scientific). After $48 \mathrm{~h}$, cells were carefully washed with PBS, total RNA was isolated with TRIzol (Invitrogen) and $1 \mu \mathrm{g}$ was converted to cDNA by RT-PCR using random hexamers $(0.5 \mu \mathrm{g}), 400$ units of MMLV-RT, $1.6 \mathrm{mM}$ total dNTPs, 20 units of Rnasin and $0.4 \mathrm{mM}$ dithiothreitol, in a $50 \mathrm{ml}$ reaction solution containing $10 \times$ RT Buffer. Before reverse transcription, RNA was treated with DNase in order to eliminate DNA contamination [33]. RT-PCR reactions were optimized for FOXC2 and GAPDH gene, in order to avoid saturation: regression curves assaying different amounts of cDNAs (corresponding to different mRNA concentrations) and different number of cycles of amplification were performed (Supplementary Figure 4). Thirty nanograms of cDNA were used to perform PCR amplification using GFP-F (5'-CGACACAATCTGCCCTTTCG-3') and FOXC2-intR (5'- CCGGGGGCGGCTCCTTG -3') primers designed to produce a $741 \mathrm{bp}$ fragment of the GFP-FOXC2 transcript. PCR conditions were as follows: denaturation at $94{ }^{\circ} \mathrm{C}$ for $10 \mathrm{~min}$, annealing at $59{ }^{\circ} \mathrm{C}$ for
$30 \mathrm{sec}$ and extension at $72{ }^{\circ} \mathrm{C}$ for $30 \mathrm{sec}$ for the first round, denaturation at $94{ }^{\circ} \mathrm{C}$ for $30 \mathrm{sec}$, annealing at $59{ }^{\circ} \mathrm{C}$ for $30 \mathrm{sec}$ and extension at $72{ }^{\circ} \mathrm{C}$ for $30 \mathrm{sec}$ for 26 cycles; denaturation at $94{ }^{\circ} \mathrm{C}$ for $30 \mathrm{sec}$, annealing at $59{ }^{\circ} \mathrm{C}$ for $30 \mathrm{sec}$ and terminal extension at $72{ }^{\circ} \mathrm{C}$ for $10 \mathrm{~min}$ for the last cycle. The PCR products were electrophoresed on a $2 \%$ agarose gel containing ethidium bromide. The concentration value of FOXC2 gene was normalized versus the constant level of GAPDH in each sample. Images of gels were acquired (Bio-Rad Gel Doc 2000, Bio-Rad, Hercules, CA, USA) and scanned using Quantity One Analysis software (Bio-Rad). This software allows the detection of the mean value of each band (i.e. the mean intensity of the pixels inside the volume of band) and the concentration of PCR products, calculated from the standards included in each gel [34].

\section{Localization assay of FOXC2 mutant proteins in HeLa and COS cells}

For transient transfections, HeLa and COS cells were cultured on glass coverslips in Dulbecco's modified Eagle's medium supplemented with $10 \%$ fetal bovine serum (FBS) and allowed to adhere overnight. The next day, the cells were transiently transfected with recombinant NT-GFP-FOXC2 plasmids using the TurboFect transfection reagent. After $24 \mathrm{~h}$, cells were fixed stained with DAPI, and examined with a Leica MB5000B microscope equipped with $40 \mathrm{X}$ and 100X Fluorart oil immersion objectives.

\section{Western blot analysis of FOXC2 proteins expression in transfected HeLa cells}

HeLa cells were transiently transfected with recombinant NT-GFP-FOXC2 plasmids as described above. Four flasks for each type of recombinant plasmids was transfected. After $48 \mathrm{~h}$, all flasks were evaluated for transfection efficiency. Only samples reaching $80 \%$ of transfection efficiency were used to prepare protein cell extracts. Cell extracts were prepared from confluent cultures grown in serum-containing medium. After extensive washing with Dulbecco's phosphate-buffered saline (DPBS), cells were recovered by scraping with a rubber policeman in $300 \mu \mathrm{l}$ of $0.05 \%$ (wt/vol) SDS. The total protein concentration of cell extracts was quantified using a Coomassie (Bradford) Protein Assay Kit (Pierce). Proteins $(10 \mu \mathrm{g} /$ well) were separated by electrophoresis on $8 \%$ SDS-polyacrylamide gel (Bio-Rad), transferred to a nitrocellulose membrane (Pierce) and then immunoblotted using a rabbit monoclonal antibody (dil. 1:5000) raised against green fluorescent protein (Invitrogen) and a mouse monoclonal antibody (dil: 1:5000) to GAPDH (Abnova). Specifically bound immunoglobulins were detected using the SuperSignal West Pico Complete Detection Kit 
(Pierce) containing ImmunoPure Peroxidase Conjugated Goat anti-Mouse and anti-Rabbit IgG (dil 1:20.000). Experiments were repeated three times.

\section{Luciferase assays}

HeLa and COS cells were co-transfected with 20 ng FOXC1 luciferase reporter [35] and 100 ng wild type or GFP-FOXC2 recombinant plasmids using TurboFect reagent (Thermo Scientific), according to the manufacturer's recommendations in a 96 well format. Each transfection was performed in triplicate. To measure FOXC2 activity, all transfected cell lines were incubated for $40 \mathrm{~h}$, prior to lysis of the culture and addition of substrate from the Britelight plus kit (PerkinElemer). For each clone, the average expression level in Fluorescent Units (FU) (from Photinus pyralis, reporter) was calculated after correction for transfection efficiency. This was obtained as measure of GFP fluorescence in the cells transfected with NT-GFP-FOXC2 recombinant plasmids [36].

The same experimental conditions were used for transient transfection of wild type or FOXC2 mutant plasmids subcloned into pcDNA3.3, a mammalian expression without tag. The dual-luciferase assays were used to obtain sequential quantification of both Photinus pyralis luciferase (reporter vector) and Renilla reniformis luciferase (control vector), as previously described (35). Luminescence detection for all transfected plasmids was performed using the Glomax luminometer (Promega). Reactions were replicated three times, using the Promega Dual Luciferase Assay kit (Promega).

\section{Statistical and bio-informatic analysis}

The statistical analysis of quantitative data of RTPCR and of Luciferase assays was made using SPSS v.19 package (SPSS, Chicago, IL, USA). The values were compared using Student's t-test. A P-value of $\leq 0.05$ was considered to be statistically significant.

The effect of amino acid substitutions on protein function was predicted using ClustalW, SIFT and PolyPhen software. A multiple sequence alignment of mammalian FOXC2 proteins was used as imput for ClustalW. The NCBI reference sequence (FOXC2 protein NCBI accession number: NP_005242.1.) of the human FOXC2 protein was used as the input for SIFT and PolyPhen, with default query options.

\section{ACKNOWLEDGMENTS}

We would like to thank Dr Luigina Illuminati, and Marco Cardone for their contribution in the clinical characterization of patients. We would like to thank Dr Elisa Colombo for luciferase assay expertise (Department of Pharmacological and Biomolecular Sciences, University of Milan, Italy).

\section{CONFLICTS OF INTEREST}

There is no conflict of interest.

\section{REFERENCES}

1. Brouillard P, Vikkula M. Genetic causes of vascular malformations. Hum Mol Genet. 2007; 16:140-149.

2. Connell F, Brice G, Jeffery S, Keele YV, Mortimer P, Smansour S. A new classification system for primary lymphatic dysplasias based on phenotype. Clin Gen. 2010; $77: 438-452$.

3. International Society of Lymphology. The diagnosis and treatment of peripheral lymphedema: 2013 Consensus Document of the International Society of Lymphology. Lymphology. 2013; 46:1-11.

4. Mendola A, Schlögel MJ, Ghalamkarpour A, Irrthum A, Nguyen HL, Fastré E, Bygum A, van der Vleuten C, Fagerberg C, Baselga E, Quere I, Mulliken JB, Boon LM, Brouillard P, Vikkula M; Lymphedema Research Group. Mutations in the VEGFR3 signaling pathway explain 36\% of familial lymphedema. Mol Syndromol. 2013; 4:257-266.

5. Brouillard P, Boon L, Vikkula M. Genetics of lymphatic anomalies. Clin Invest. 2014; 124:898-904.

6. Fang J, Dagenais SL, Erickson RP, Arlt MF, Glynn MW, Gorski JL, Seaver LH, Glover TW. Mutations in FOXC2 (MFH-1), a forkhead family transcription factor, are responsible for the hereditary Lymphedema-Distichiasis Syndrome. Am J Hum Genet. 2000; 67:1382-1388.

7. Bell R, Brice G, Child AH, Murday VA, Mansour S, Sandy CJ, Collin JRO, Brady AF, Callen DF, Burnand K, Mortimer P, Jeffery S. Analysis of lymphedema-distichiasis families for FOXC2 mutations reveals small insertions and deletions throughout the gene. Hum Genet. 2001; 108:546551.

8. Brice G, Mansour S, Bell R, Collin JRO, Child AH, Brady AF, Sarfarazi M, Burnand KG, Jeffery S, Mortimer P, Murday VA. Analysis of the phenotypic abnormalities in Lymphoedema-distichiasis syndrome in 74 patients with FOXC2 mutations or linkage to 16q24. J Med Genet. 2002; 39:478-483.

9. Sabine A, Petrova TV. Interplay of mechanotransduction, FOXC2, connexins, and calcineurin signaling in lymphatic valve formation. Adv Anat Embryol Cell Biol. 2014; 214:67-80

10. Danciu TE, Chupreta S, Cruz O, Fox JE, Whitman M, Iñiguez-Lluhí JA. Small ubiquitin-like modifier (SUMO) modification mediates function of the inhibitory domains of developmental regulators FOXC1 and FOXC2. J Biol Chem. 2012; 287:18318-18329.

11. Ivanov KI, Agalarov Y, Valmu L, Samuilova O, Liebl 
J, Houhou N, Maby-El Hajjami H, Norrmén C, Jaquet M, Miura N, Zangger N, Ylä-Herttuala S, Delorenzi M, Petrova TV. Phosphorylation regulates FOXC2-mediated transcription in lymphatic endothelial cells. Mol Cell Biol. 2013; 33:3749-3761.

12. Lam EWF, Brosens JJ, Gomes AR, Koo CY. Forkhead box proteins: tuning forks for transcriptional harmony. Nature. $2013 ; 13,: 482-495$.

13. Kriederman BM, Myloyde TL, Witte MH, Dagenais SL, Witte CL, Rennels M, Bernas MJ, Lynch MT, Erickson RP, Caulder MS, Miura N, Jackson D, Brooks BP, Glover TW. FOXC2 haploinsufficient mice are a model for human autosomal dominant lymphedema-distichiasis syndrome. Hum Mol Genet. 2003; 12:1179-1185.

14. Petrova TV, Karpanen T, Norrmén C, Mellor R, Tamakoshi T, Finegold D, Ferrell R, Kerjaschki D, Mortimer P, YläHerttuala S, Miura N, Alitalo K. Defective valves and abnormal mural cell recruitment underlie lymphatic vascular failure in lymphedema distichiasis. Nature Medicine. 2004; 10:974-981.

15. Iida K, Koseki H, Kakinuma H, Kato N, Mizutani-Koseki Y, Ohucki H, Yoshioka H, Noji S, Kawamura K, Kataoka Y, Ueno F, Taniguchi M, Yoshida N, Sugiyama T, Miura $\mathrm{N}$. Essential roles of the winged helix transcription factor MFH-1 in aortic arch patterning and skeletogenesis. Development. 1997; 124:4627-4638.

16. Winnier GE, Hargett L, Hogan BL. The winged helix transcription factor MFH1 is required for proliferation and patterning of paraxial mesoderm in the mouse embryo. Genes Dev. 1997; 11:926-940.

17. van Steensel MA, Damstra RJ, Heitink MV, Bladergroen RS, Veraart J, Steijlen PM, van Geel M. Novel missense mutations in the FOXC2 gene alter transcriptional activity. Hum Mutat. 2009; 30:E1002-1009.

18. Berry FB, Tamini Y, Carle MV, Lehmann OJ, Walter MA. The establishment of a predictive mutational model of the forkhead domain through the analyses of FOXC2 missense mutations identified in patients with hereditary lymphedema with distichiasis. Hum Mol Genet. 2005; 14:2619-2627.

19. Michelini S, Degiorgio D, Cestari M, Corda D, Ricci M, Cardone M, Mander A, Famoso L, Contini E, Serrani R, Pinelli L, Cecchin S, Bertelli M. Clinical and genetic study of 46 italian patients with primary lymphoedema. Lymphology. 2012; 45:3-12.

20. Finegold DN, Kimak MA, Lawrence EC, Levinson KL, Cherniske EM, Pober BR, Dunlap JW, Ferrel RE. Truncating mutations in FOXC2 cause multiple lymphedema syndromes. Hum Mol Genet. 2001; 10:11851189.

21. Bahuau M, Houndayer C, Tredano M, Soupre V, Couderc $\mathrm{R}$, Vazquez MP. FOXC2 truncating mutation in distichiasis, lymphedema, and cleft palate. Clin Genet. 2002; 62:470473.
22. Sholto-Douglas-Vernon C, Bell R, Brice G, Mansour S, Sarfarazi M, Child AH, Smith A, Mellor R, Burnand K, Mortimer P, Jeffery S. Lymphoedema-distichiasis and FOXC2: unreported mutations, de novo mutation estimate, families without coding mutations. Hum Genet. 2005; 117:238-242.

23. Castellana S, Mazza T. Congruency in the prediction of pathogenic missense mutations: state-of -the-art web-based tools. Brief Bioinform. 2013; 14:448-459.

24. Richard E, Douillard-Guilloux G, Caillaud C. New insights into therapeutic options for Pompe disease. IUBMB Life. 2011; 63:979-986.

25. Kampmann C, Kalkum G, Beck M, Whybra C. Successful long-term enzyme replacement therapy in a young adult with Fabry disease. Clin Genet. 2013; 83:395-396.

26. Tavian D, Missaglia S, Redaelli C, Pennisi EM, Invernici G, Wessalowski R, Maiwald R, Arca M, Coleman RA. Contribution of novel ATGL missense mutations to the clinical phenotype of NLSD-M: a strikingly low amount of lipase activity may preserve cardiac function. Hum Mol Genet. 2012; 15:5318-28.

27. Missaglia S, Tasca E, Angelini C, Moro L, Tavian D. Novel missense mutations in PNPLA2 causing late onset and clinical heterogeneity of neutral lipid storage disease with myopathy in three siblings. Mol Genet Metab. 2015; 115:110-117.

28. Erikson RP, Dagenais SL, Caulder MS, Downs CA, Herman G, Jones MC, Kerstiens-Frederikse WS, Lidral AC, McDonalds M, Nelson CC, Witte M, Glover TW. Clinical heterogeneity in lymphedema-distichiasis with FOXC2 truncating mutations. J Med Genet. 2001; 38:761-766.

29. Koltowska K, Betterman KL, Harvey NL, Hogan BM. Getting out and about: the emergence and morphogenesis of the vertebrate lymphatic vasculature. Development. 2013; 140:1857-1870.

30. Park C, Kim TM, Malik AB. Transcriptional regulation of endothelial cell and vascular development. Circ Res. 2013; 112:1380-400.

31. Cui YM, Jiao HL, Ye YP, Chen CM, Wang JX, Tang N, Li TT, Lin J, Qi L, Wu P, Wang SY, He MR, Liang L, Bian XW, Liao WT, Ding YQ. FOXC2 promotes colorectal cancer metastasis by directly targeting MET. Oncogene. 2015; 34:4379-4390.

32. Paranjape AN, Soundararajan R, Werden SJ, Josph R, Taube JH, Liu H, Rodriguez-Canales, Sphyris N, Witsuba I, Miura N, Dhillon J, Mahajan K, Chang JT, Ittman M, Maity SN, Logothetis C, Tang TG, Mani SA. Inhibition of FOXC2 restores epithelial phenotype and drug sensitivity in prostate cancer cells with stem-cell properties. Oncogene. 2016; 1-14.

33. Tavian D, Roncaglia N, Vergani P, Cameroni I, Colombo R, Coviello DA. A new splicing site mutation of the ABCB4 gene in intrahepatic cholestasis of pregnancy with raised 
serum $\gamma$-GT. Dig Liver Dis. 2009; 41:671-675.

34. Tavian D, De Giorgio A, Granato A. Selective underexpression of Kv3.2 and Kv3.4 channels in the cortex of rats exposed to ethanol during early postnatal life. Neurol Sci. 2011; 32:571-577.

35. Saleem RA, Banerjee-Basu S, Berry FB, Baxevanis AD, Walter MA. (2001) Analyses of the effects that diseasecausing missense mutations have on the structure and function of the winged-helix protein FOXC1. Am J Hum Genet. 2001; 68:627-641.
36. Manente AG, Pinton G, Tavian D, Lopez-Rodas G, Brunelli E, Moro L. Coordinate sumoylation and ubiquitination modulate EGF induced EGR1 expression and stability. PLoS One. 2011; 6:e25676. 